\title{
http://bjas.journals.ekb.eg \\ Comparative Study between Kirschner Wires and Mini-Set Plate in Management of Unstable Metacarpal Shaft Fractures
}

M.S.Shawky, W.A.Kandel, A.M.Halawa and A.I.Mansour

Orthopedic Surgery, Faculty of Medicine, Benha Univ., Benha, Egypt

E-Mail:midonazy@gmail.com

\begin{abstract}
Metacarpal fractures comprise between $18-44 \%$ of all hand fractures. Non-thumb metacarpals account for around $88 \%$ of all metacarpal fractures, with the fifth finger most commonly involved. This is a comparative study between Kirschner wires and mini-set plate in management of unstable metacarpal shaft fractures. This is a review article, The search was performed in MEDLINE, Embase, Pubmed and CINAHL Plus in the same date range with the following mediacl terms: "Kirschner wires; mini-set plate; unstable; metacarpal shaft; fractures.", including articles from 2000 to 2019, K-wire fixation is simple to manipulate and without or with little damage on fracture blood supply, and has been used extensively. Since in the K-wire fixation there is no compression on the fracture, it is difficult to control the motions of the fingers. Stiffness resulting from tendon adhesions and joint contractures is the most common complication associated with hand fractures. Conversely, fractures that require mini-plate fixation but are treated without fixation or with inadequate fixation still lead to stiffness and are associated with an increased rate of nonunion and malunion. Kirschner wires are almost always used for temporary fixation and are removed after the early appearance of fracture callus on radiographs. Despite the availability of many options for fixation, percutaneous fixation using Kirschner wires is a good method for treatment of unstable ulnar four metacarpal fractures. We recommended this technique, which is more biological, technically easier, safe, cheap and rapid to perform with good functional outcome.
\end{abstract}

Keywords: Kirschner wires, Mini-set plate, Unstable, Metacarpal shaft, Fractures.

\section{Introduction}

The metacarpal bone is made out of three sections: head, shaft and base. The head and the base are made inside out of cancellous bone encompassed by a moderately meager cortical shell. The pole made out of thicker cortical bone that encloses the open medullary channel. The metacarpal bones are short long bones that lucid proximally with the carpal bone by synovial ellipsoidal joints for the average for fingers and seat joint for the thumb. The metacarpal bones articulate with the proximal phalanx of the finger by condyloid joints [1].

Hand wounds are exceptionally regular coming about every now and again in metacarpal and phalangeal cracks [2].

Metacarpal cracks contain between $18-44 \%$ of all hand breaks. Non-thumb metacarpals represent around $88 \%$ of every metacarpal break, with the fifth finger most usually included. Most metacarpal cracks happen in the dynamic and working populace, especially teenagers and youthful grown-ups and as a rule result from a hard impact, pulverize or rocket injury [3].

Most hand breaks are steady either previously or after shut decrease and can be successfully rewarded by shut methods. Albeit numerous metacarpal and phalangeal breaks might be dealt with non-operatively, employable treatment might be demonstrated in the setting of different cracks, over the top shortening, rotational distortion or inordinate rakish deformation. Obsession decisions rely upon the break design, specialist inclination and related wounds [4].

Fitting treatment incorporates satisfactory evaluation, physical assessment, and coordinated imaging. Such a methodology should prompt a sane arrangement that centers around the recovery of every single harmed part including rigid, articular, and delicate tissue structures. Numerous careful choices have been depicted to treat metacarpal shaft breaks with no accord on ideal strategy. Choices incorporate open decrease inside obsession, Kirschner (K-) wire obsession, stitch obsession, cerclage wiring, scaled down plate and smaller than normal screws [5].

Insignificantly obtrusive procedures with Kirschner wires is an alluring choice, as broad careful analyzation delicate tissue devitalization at the break site can be stayed away from. It additionally restricts the likely intricacy of extensor aggravation by a dorsalplate, diminishing the opportunity of extensor tenosynovitis, despite the fact that this complexity has still been accounted for with K-wire obsession [6].

The hindrances of the wire procedure are absence of supreme dependability, wire movement, pin site issues, contamination and the need for embed expulsion, extensor tenosynovitis and tactile unsettling influences [7].

This is a similar report between Kirschner wires and smaller than expected set plate in the executives of insecure metacarpal shaft breaks

\section{Methods}

This is an audit article, The hunt was acted in MEDLINE, Embase, Pubmed and CINAHL Plus in a similar date run with the accompanying mediacl terms: "Kirschner wires; smaller than normal set plate; shaky; metacarpal shaft; fractures.",including articles from 2000 to 2019, Excluded articles from survey are those of langauge other than English. Watchwords: Kirschner wires; smaller than expected set plate; insecure; metacarpal shaft; breaks.

\section{Results}

The decision of plating versus percutaneous $\mathrm{k}$ wire obsession might be made dependent on crack qualities, related wounds or specialist inclination. It additionally 
might be identified with the geographic area of the medical procedure, level of emergency clinic framework or the time that the case happens. As inserts for ORIF of metacarpal break have developed and improved, the signs have expanded and the achievement rate has expanded for these systems.

Plating conveys with it a high pace of difficulties, while k-wiringlacks the innate dependability of plating, especially with the appearance of locking screws. In any case, handily positioned percutaneous $\mathrm{k}$ wires in numerous planes can give adequate security to allow early dynamic helped movement.

$\mathrm{K}$-wire obsession is easy to control and without or with little harm on crack blood flexibly, and has been usedextensively. Since in the K-wire obsession there is no pressure on the crack, it is hard to control the movements of the fingers.

Solidness coming about because of ligament bonds and joint contractures is the most well-known intricacy related with hand breaks. On the other hand, breaks that require smaller than usual plate obsession yet are treated without obsession or with lacking obsession despite everything lead to firmness and are related with an expanded pace of nonunion and malunion.

Kirschner wires are quite often utilized for impermanent obsession and are evacuated after the early appearance of break callus on radiographs.

Small scale screws and plates are normally just evacuated for differentreasons incorporate unmistakable quality and aggravation under the skin, relaxing, pullout, or breakage. Slackening or breakage may proclaim postponed association, nonunion, or malunion. Inserts may likewise be evacuated at the hour of tenolysis or capsulotomy.

\section{Classification of Metacarpal Fracture AO-OTA Classification}

The Orthopedic Trauma Association classification uses an alpha-numerical classification scheme, Fig (1).

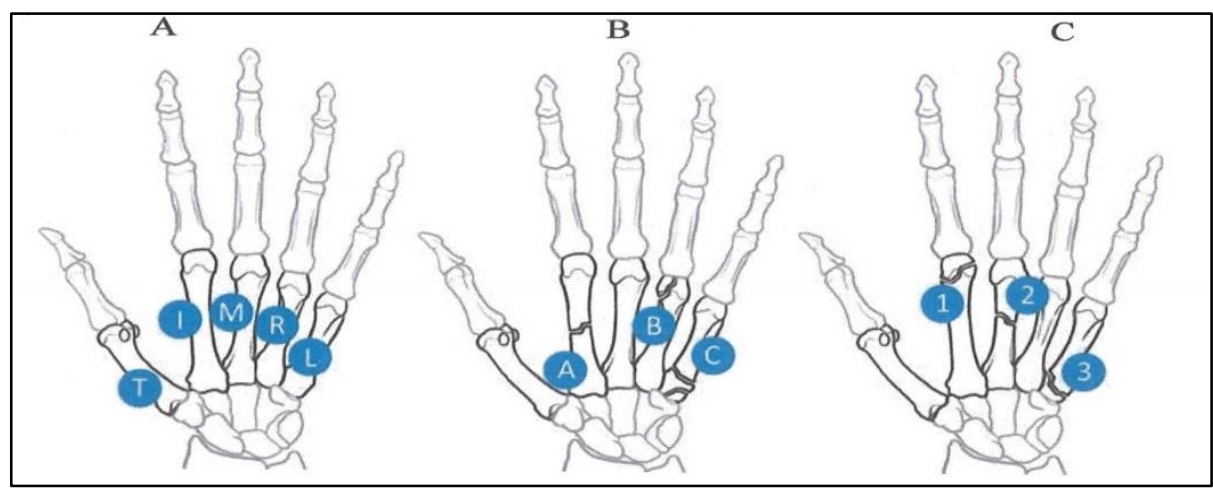

Fig (1) The OTA classification of metacarpal fractures with (A) modifiers as which digit is involved ( $\mathrm{T}$ thumb, I index, $\mathrm{M}$ middle, $\mathrm{R}$ ring, $\mathrm{L}$ little), (B) the articular involvement (A extra-articular, B articular, C articular/extra-articular), (C) the bony location of the fracture (1 head, 2 shaft, 3 base) [8].

A functional classification should take into account fracture location, pattern, associated soft tissue injury, deformity and intrinsic stability.

Table (1) Functional Fracture Parameters: Metacarpal Fracture Characteristics [9].

\begin{tabular}{|c|c|c|c|c|c|c|}
\hline Location & Pattern & Skeleton & Deformity & $\begin{array}{c}\text { Soft } \\
\text { tissue }\end{array}$ & $\begin{array}{c}\text { Associated } \\
\text { injury }\end{array}$ & $\begin{array}{c}\text { Reaction to } \\
\text { motion }\end{array}$ \\
\hline Base & Transverse & Simple & Angulation & Closed & Skin & Stable \\
\hline Shaft & Oblique & Impacted & $\begin{array}{c}\text { Dorsal } \\
\text { displacement }\end{array}$ & Open & Tendon & Unstable \\
\hline Neck & Spiral & Comminuted & $\begin{array}{c}\text { Dorsal } \\
\text { displacement }\end{array}$ & & Ligament & - \\
\hline $\begin{array}{l}\text { Head (condyle ) } \\
\text { epiphysis }\end{array}$ & Avulsion & Bone loss & $\begin{array}{l}\text { Rotation } \\
\text { Shortening }\end{array}$ & $\bar{Z}$ & $\begin{array}{c}\text { Nerve } \\
\text { Blood vessel }\end{array}$ & 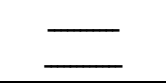 \\
\hline
\end{tabular}

Proper treatment incorporates satisfactory evaluation, physical assessment, and coordinated imaging. Such a methodology should prompt an objective arrangement that centers around the recovery of every harmed part, including rigid, articular, and delicate tissue structures. Numerous careful choices have been depicted to treat metacarpal shaft breaks with no accord on ideal method. Alternatives incorporate open decrease interior obsession,
Kirschner (K-) wire obsession, stitch obsession, cerclage wiring, smaller than normal plate and scaled down screws.

Insignificantly intrusive strategies with Kirschner wires is an appealing alternative, as broad careful dismemberment delicate tissue devitalization at the crack site can be kept away from. It additionally restricts the possible confusion of extensor disturbance by a dorsal 
plate, decreasing the opportunity of extensor tenosynovitis, despite the fact that this intricacy has still been accounted for with K-wire obsession.

The inconveniences of the wire method are absence of outright security, wire movement, pin site issues, disease and the need for embed expulsion, extensor tenosynovitis and tangible aggravations.

\section{Discussion}

Cracks of the metacarpals are very normal, adding up to around $33 \%$ of all hand breaks. A lion's share of these cracks are shut, basic, transverse, or short slanted and are agreeable to defensive splintage and early movement [10].

Most cases can be dealt with moderate strategies after shut decrease; in any case, careful mediation is shown when an unsuitable decrease continues, a large portion of the writing concurred that any level of rotational deformation and shortening of more prominent than $5 \mathrm{~mm}$ is a sign for careful treatment [11].

Choice of the ideal treatment relies upon numerous components, including break area (intra-articular versus extra-articular), crack geometry (transverse, winding or diagonal, comminuted), disfigurement (precise, rotational, shortening), regardless of whether the break is open or shut, whether rigid and delicate tissue wounds are related and inherent crack solidness. Notwithstanding the favored method of treatment, the objective ought to be full and quick rebuilding of capacity [12].

Metacarpal shortening and malrotation are critical components in the last clinical result in any damaged hand. Shortening of the metacarpal by as much as $2 \mathrm{~mm}$ prompts an extensor slack of $7^{\circ}$ and a $8 \%$ hold quality misfortune. Additionally rotational deformation of the metacarpals is ineffectively endured as the pivoted finger will in general get encroached or covered with different fingers [10].

Different surgery have been portrayed for treatment of metacarpal shaft breaks including $\mathrm{K}$-wires, circulage wires, intramedullary nails, pressure band wires, slack screws, plating and outside fixators. Of these strategies $\mathrm{K}$-wires obsession is a mainstream decision because of its straightforwardness and insignificant delicate tissue taking care of. Along these lines, numerous procedures have been depicted for obsession utilizing K-wires, for example, crossing wires, intramedullary antegrade and retrograde wires addition, transverse wiring and Bouquet osteosynthesis [13].

Different techniques for inward obsession in metacarpal cracks exist, the standards of treatment incorporate reclamation of articular life systems, stable obsession of breaks, end of rakish or rotational disfigurement and fast rebuilding of portability and capacity [14].

There have been numerous reports of issues with utilizing plating for these breaks, for example, introduction of the crack site to be specific piece devascularization and delicate tissue disturbance, solidness is likewise a significant issue and these plates cause noteworthy osteopenia. Additionally the relationship of investigation of inner obsession suggests routine evacuation of these plates at around a half year after medical procedure which involves another surgery [10].

Meyer et al., audited 81 patient with metacarpal cracks rewarded with plate and screw, Twenty-eight of 81 patients $(35 \%)$ and 33 of 104 breaks $(32 \%)$ had at least one difficulties. 15 of 81 patients $(19 \%)$ and 20 of 104 breaks $(19 \%)$ had at least one significant inconvenience. Thirteen of 81 patients $(16 \%)$ and 13 of 104 breaks $(13 \%)$ had at least one minor entanglement. Twelve patients $(15 \%)$ experienced trouble with crack mending. There were six deferred associations and six nonunion. One patient with a suggestive postponed association and each of the six patients with a nonunion experienced extra medical procedure. Eight patients (10\%) experienced practically significant solidness with a TAM 180 degrees, and five required a subsequent activity to improve scope of movement. Plate extricating or breakage happened in seven patients (8\%), and equipment evacuation was vital in two patients who were indicative [15].

Page and Stern looked into 66 metacarpal cracks rewarded with plates and screws uncovered a $36 \%$ complexity rate. Solidness was the most usually revealed inconvenience, with $76 \%$ of patients considered answered to have complete dynamic movement under $220^{\circ}$. Sixteen percent of intricacies revealed included a minor extensor slack, while $7.9 \%$ had contractures, trailed by significant extensor slack in $6.3 \%$ of complexities. Progressively genuine difficulties are uncommon, with nonunion, contamination and ligament crack each containing 1.6 $\%$ of intricacies announced [14].

Correction medical procedure because of equipment confusion has been accounted for at paces of 4.6-32\% [16].

In 2018 Lorenzo et al., distributed their outcome more than 150 patients rewarded with antegrade intramedullary K-wires for obsession of metacarpal cracks, the mean term of medical procedure was 14 min ( run 4 - $33 \mathrm{~min}$ ), Q-DASH score indicated a normal estimation of 12.3 focuses (run, 0 to 37 focuses), Bone association was seen in every patient after an interim of 4.7 weeks ( 4 to $7 \mathrm{wk}$ ). Rotational distortion was seen in 8 patients, An aggregate of 12 patients built up a shallow pin site disease/irritation, all of which settled after treatment with oral anti-infection agents [12].

In 2013 Lee et al., oversaw 56 patients with metacarpal cracks with retrograde intramedullary nail. The normal follow-up term was 13 months. Radiographic bone association was accomplished in all patients, with a mean mending time of 5.2 weeks (go, 5-7 weeks). Four patients created extensor ligament aggravation during development, yet the bothering vanished totally after evacuation of the wires. Three patients built up a shallow pin site contamination, all of which settled after wire evacuation and treatment with 
oral anti-infection agents. One patient demonstrated deadness in the region of the dorsal tactile part of the ulnar nerve, which settled following 4 months without explicit treatment. Five patients had some solidness [17].

In 2013 Potenza et al., utilized transverse wiring strategy in the board of 35 cases with metacarpal breaks. At about two months post-employable association was accomplished in practically all patients. In five cases, a minor neighborhood contamination happened at the site of presentation of the K-wires, and it was dealt with effectively with anti-microbial treatment. In two patients, a slight impediment of the expansion of the fifth MP joint of under 108 was watched, in spite of the fact that they didn't report any noteworthy hindrance of their hand work. Leftover palmar angulation of the top of the fifth metacarpal was found in three patients, averaging 78 (territory 5-138) [18].

In 2007 Choi and Song distributed the aftereffects of their investigation utilizing transverse $\mathrm{K}$-wires for obsession of metacarpal cracks in 34 patients. Three cases joined with angulation more than 20 degrees; five cases had skin issues around the tips of the K-wires. Three cases had remaining rotational disfigurement. One case was not joined together and the $\mathrm{K}$-wires were expelled and rewarded by plate and screws [19].

Bunch osteosynthesis (different intramedullary adaptable wires obsession for metacarpal breaks) had excellent outcome in the course of the most recent two decades. This strategy comprises of an open procedure anterograde intramedullary obsession with three pretwisted Kirschner wires embedded into the diminished metacarpal head in disparate ways [6].

Fusetti et al., in 2002 recommended that the upside of the percutaneous intramedullary K-wires obsession in treatment of unsteady metacarpal cracks is the shirking of the inconvenience that happen with open decrease and inside obsession, for example, troubles with the break mending, disease, firmness because of broad delicate tissue dismemberment, later fibrosis and extensor ligament attachment, extricating of the plate [15].

Foucher, played out his investigation more than 66 patients with flimsy metacarpal breaks rewarded by antegrade intramedullary sticking. All patients came back to their typical action with ordinary quality in 61 patients and diminished by $11 \%$ in the rest of the cases. Postoperative complexities were rare and included one instance of reflex thoughtful dystrophy (with no remaining disability) and one instance of dorsal ulnar tactile neuritis. There were no hematomas, diseases, auxiliary crack removals, no extensor ligament breaks. Ten patients required a powerful flexion brace to improve the inactive scope of movement of the MP joint [6].

This was in accordance with the aftereffect of Sletten et al. in 2014 who performed bundle sticking in 41 patients with flimsy metacarpal breaks. The mean TAM was $260^{\circ}$ with hold quality $(\mathrm{kg}) 49$. The general VAS fulfillment (0-100, 100 best) was 100 and mean
Quick DASH score $(0-100,0$ best) was 0 running from (0 - 41). Just two patients created shallow pin tract disease whom made do with oral anti-microbials and 2 cases experienced pin movement. 4 patient had gentle rotational deformation at 1 year development however it was not hindering the practical result and not required careful mediation [20].

Amsallem et al., in 2018 distributed their investigation where 30 patients with fighter's break made do with rearranged bunch strategy utilizing single thick K-wire. The mean usable time was 14 min (7-28 min). Following 3 months line up all cracks recuperated with mean TAM $264^{\circ}$. No disease, 2ry uprooting, Kwire movement nor injury to tangible part of ulnar nerve was prove [21].

This was better than the consequences of Mohammed et al., who updated one break for bombed obsession and revealed 3 shallow pin tract contamination and two patient had mellow extensor slack of about $15^{\circ}$ [2].

In 2007 Winter et al., played out a forthcoming randomized controlled preliminary, the investigation showed that 18 patient worked with antegrade bundle sticking had $\mathrm{s}$ marginally better complete dynamic movement (TAM) of the fifth finger and better ROM in the fifth MP joint than 18 patient rewarded with transverse sticking at definite short follow-up following 3 months. The distinctions were just 18 and $12^{\circ}$ separately [22].

This was in accordance with the aftereffects of the review study done by Schädel-Höpfiner and collaborators in 2007 demonstrated an essentially better result at year and a half follow-up after antegrade intramedullary sticking in 15 patients contrasted with retrograde crossed sticking in 15 patient in regards to torment and ROM in the MP joint. No noteworthy contrasts in hold quality and DASH score [23].

Sletten et al., in 2014 made a review concentrate more than 67 patients contrasting $b$.

\section{Conclusion}

Despite the availability of many options for fixation, percutaneous fixation using Kirschner wires is a good method for treatment of unstable ulnar four metacarpal fractures. We recommended this technique, which is more biological, technically easier, safe, cheap and rapid to perform with good functional outcome.

\section{References}

[1] L. W. Catalano, D. A. Zlotolow, M. P. Lafer, "Surgical exposures of the wrist and hand," JAAOS-Journal Am. Acad. Orthop. Surg, Vol. 20(1), PP. 48-57, 2012.

[2] R. Mohammed, M. Z. Farook, K. Newman, "Percutaneous elastic intramedullary nailing of metacarpal fractures: surgical technique and clinical results study," J. Orthop. Surg. Res, Vol. 6(1), PP. $1-5,2011$.

[3] K. M. Kollitz, W. C. Hammert, N. B. Vedder, "Metacarpal fractures: treatment and 
complications," Hand, Vol. 9(1), PP. 16-23, 2014.

[4] J. E. Adams, T. Miller, M. Rizzo, "The biomechanics of fixation techniques for hand fractures.," Hand Clin., Vol. 29(4), PP. 493-500, 2013.

[5] E. Itadera, R. Hiwatari, H. Moriya, "Closed intramedullary fixation for metacarpal fractures using J-shaped nail," Hand Sur, Vol. 13(3), PP. 139-145, 2008.

[6] G. Foucher, "'Bouquet' osteosynthesis in metacarpal neck fractures: a series of 66 patients," J. Hand Surg. Am., Vol. 20(3), PP. S86-S90, 1995.

[7] L. S. Zhang, Y. W. Pan, G. L. Tian, "Biomechanical research of antegrade intramedullary fixation for the metacarpal fractures," Zhonghua Wai Ke Za Zhi, Vol. 48(8), PP. 606-609, 2010.

[8] A. Speeckaert,H. M. Awan, "First Metacarpal Base Fractures," in Orthopedic Surgery Clerkship, Springer, Vol. 2(2), PP. 179-181, 2017.

[9] S. R. Haj Zargarbashi, B. Panjavi, H. R. A. Hamid Reza Arti, "Management of Metacarpal Nonunion in a Two-Year-Old Child," Trauma Mon, Vol. 24(1), PP. 1-3, 2019.

[10] M. V Agashe, S. Phadke, V. M. Agashe, "A new technique of locked, flexible intramedullary nailing of spiral and comminuted fractures of the metacarpals: a series of 21 cases," Hand, Vol. 6(4), PP. 408-415, 2011.

[11]S. Facca, R. Ramdhian, A. Pelissier, "Fifth metacarpal neck fracture fixation: locking plate versus K-wire?," Orthop. Traumatol. Surg. Res, Vol. 96(5), PP. 506-512, 2010.

[12] L. Rocchi, G. Merendi, L. Mingarelli, “Antegrade percutaneous intramedullary fixation technique for metacarpal fractures: prospective study on 150 cases," Tech. Hand Up. Extrem. Surg, Vol. 22(3), PP. 104-109, 2018.

[13] C. McCarthy, J. B. Samora, H. Awan, "Metacarpal shaft fractures: A review," OA Orthop, Vol. 2(2), p. 12, 2014.

[14] S. M. Page , P. J. Stern, "Complications and range of motion following plate fixation of metacarpal and phalangeal fractures," J. Hand Surg. Am, Vol. 23(5), PP. 827-832, 1998.

[15]C. Fusetti, H. Meyer, N. Borisch, "Complications of plate fixation in metacarpal fractures," J. Trauma Acute Care Surg, Vol. 52(3), PP. 535-539, 2002.

[16]E. Melamed, L. Joo, E. Lin, "Plate fixation versus percutaneous pinning for unstable metacarpal fractures: a meta-analysis," J. Hand Surg. (AsianPacific Vol, Vol. 22(1), PP. 29-34, 2017.

[17] S. K. Lee, K. J. Kim, W. S. Choy, "Modified retrograde percutaneous intramedullary multiple Kirschner wire fixation for treatment of unstable displaced metacarpal neck and shaft fractures," Eur. J. Orthop. Surg. Traumatol, Vol. 23(5), PP. 535543, 2013.

[18] V. Potenza, R. Caterini, F. De Maio, S "Fractures of the neck of the fifth metacarpal bone. Mediumterm results in 28 cases treated by percutaneous transverse pinning," Injury, Vol. 43(2), PP. 242245, 2012.

[19] N. Y. Choi , H. S. Song, "Treatment of Metacarpal Fractures using Transverse Kirschner-wire Fixation," J. Korean Orthop. Assoc, Vol. 42(5), PP. 608-615, 2007.

[20] I. N. Sletten, J. C. Hellund, B. Olsen, “Conservative treatment has comparable outcome with bouquet pinning of little finger metacarpal neck fractures: a multicentre randomized controlled study of 85 patients," J. Hand Surg, Vol., Vol. 40(1), PP. 7683, 2015.

[21]L. Amsallem "'Simplified internal fixation of fifth metacarpal neck fractures," Orthop. Traumatol. Surg. Res, Vol. 104(2), PP. 257-260, 2018.

[22] M. Winter, T. Balaguer, C. Bessiere, "Surgical treatment of the boxer's fracture: transverse pinning versus intramedullary pinning," J. Hand Surg, Vol. 32, no. 6, PP. 709-713, 2007.

[23]M. Schädel-Höpfner, M. Wild, J. Windolf, "Antegrade intramedullary splinting or percutaneous retrograde crossed pinning for displaced neck fractures of the fifth metacarpal?," Arch. Orthop. Trauma Surg, Vol. 127(6), PP. 435440, 2007.

[24]R. Fujitani, S. Omokawa, K. Shigematsu, "Comparison of the intramedullary nail and lowprofile plate for unstable metacarpal neck fractures," J. Orthop. Sci , Vol. 17(4), PP. 450-456, 2012. 